\title{
Uniqueness of an inverse problem for an integro-differential equation related to the Basset problem
}

Bin $\mathrm{Wu}^{1 *}$ and Jun $\mathrm{Yu}^{2}$

"Correspondence:
binwu@nuist.edu.cn
"School of Mathematics and
Statistics, Nanjing University of
Information Science and
Technology, Nanjing, 210044, China
Full list of author information is
available at the end of the article

\begin{abstract}
This paper concerns an inverse problem for an integro-differential equation related to the Basset problem. The inverse problem aims to determine a weakly singular term from the time trace at a fixed point $x_{0} \in \Omega$. We use the maximum principle for an integro-differential operator to derive the uniqueness of the inverse problem. Additionally, we prove the existence and uniqueness of the direct Basset problem with a general kernel function.
\end{abstract}

MSC: 35L05; 35L10; 35R09; 35R30

Keywords: inverse problems; Basset problem; uniqueness

\section{Introduction}

Let $\Omega \subset \mathbb{R}^{N}(N=1,2,3)$ be a bounded domain with smooth boundary $\partial \Omega:=\Gamma$. We further set $Q_{0, T}:=\Omega \times(0, T), \Sigma_{0, T}=\Gamma \times(0, T)$. Then we consider the following integrodifferential equation $[1-3]$ :

$$
L_{0, t}[u](x, t):=u_{t}(x, t)-A[u](x, t)+\int_{0}^{t} k(t-s) u_{t}(x, s) \mathrm{d} s=f(x, t), \quad(x, t) \in Q_{0, T}
$$

with the initial and boundary conditions:

$$
\begin{cases}u(x, 0)=u_{0}(x), & x \in \Omega, \\ u(x, t)=g(x, t), & (x, t) \in \Sigma_{0, T} .\end{cases}
$$

Here the operator $A$ is uniformly elliptic on $\bar{\Omega}$, defined by

$$
A[u](x, t)=\frac{\partial}{\partial x}\left(a(x) \frac{\partial}{\partial x} u(x, t)\right), \quad(x, t) \in Q_{0, T},
$$

with $a(x) \in C^{1}(\bar{\Omega})$ satisfying $a(x) \geq a_{0}>0$ for $x \in \bar{\Omega}$, the function $k$ with some power singularity is unknown and has to be determined by the following measurement data at a fixed point $x_{0} \in \Omega$ :

$$
u\left(x_{0}, t\right)=h(t), \quad t \in[0, T] .
$$

\section{Springer}

O 2014 Wu and Yu; licensee Springer. This is an Open Access article distributed under the terms of the Creative Commons Attribution License (http://creativecommons.org/licenses/by/4.0), which permits unrestricted use, distribution, and reproduction in any medium, provided the original work is properly credited. 
For the direct problem (1.1) and (1.2), Ashyralyev [1] proved the well-posedness for the following form of $k$ :

$$
k(t)=\frac{1}{\Gamma(1-\alpha)} t^{-1 / 2} .
$$

In this case, (1.1) can be written as a fractional parabolic equation

$$
u_{t}(x, t)-A u(x, t)+\partial_{t}^{\frac{1}{2}} u(x, t)=f(x, t) .
$$

For details on the fractional derivative, we refer the readers to [4] or [5]. The system (1.1) and (1.2) is called the Basset problem, which describes a classical problem in fluid dynamics with the unsteady motion of a particle accelerates in a viscous fluid due to the force of gravity [6]. For the recent results on the Basset problem, we refer the readers to [7-12]. In Appendix A, we will prove the existence and uniqueness of the direct problem (1.1) and (1.2) for general $k$, which extends the results in [1] and [2]. (References [1] and [2] were concerned the direct Basset problem with the fractional order 1/2, i.e. the problem (1.4) and (1.2).) For the other models related to the integro-differential equation, we refer the readers to [13-15].

As for the inverse kernel problems related to the integro-differential equation, in [1619] efficient strategies to prove the existence and uniqueness of inverse memory kernel problems were given. In particular, Colombo and Guidetti [16] showed that a semilinear integro-differential parabolic inverse problem had a unique solution global in time under suitable growth conditions for the nonlinearity involved in the evolution equation. Lorenzi and Rocca [18] studied an inverse two memory kernels problem in a hyperbolic phase-field model. But the additional measurements used in these papers are imposed on the whole or the part of $\Omega$, which can be expressed in the following integral form:

$$
\int_{\Omega} \phi(x) u(x, t) \mathrm{d} t=g(t), \quad t \in[0, T]
$$

with a known function $\phi$. Comparing with these papers, our study only needs the measurements at a fixed point $x_{0}$. Another difference is that the kernel function discussed in our current paper has power singularity $t^{-\gamma}$. Finally, our method in discussing the uniqueness of our inverse problem is different from those inverse memory kernel problems, in which the methods on the basis of the analytic semigroup theory were used. It is worth noting that in [20] the Fourier method was applied to prove the existence and uniqueness of determining a weakly singular kernel in a linear heat conduction model.

For some $\gamma \in(0,1)$, we use the notation $C_{\gamma}[0, T]$ to denote the following Banach space: $C_{\gamma}[0, T]:=\left\{k \mid t^{\gamma} k \in C[0, T]\right\}$, endowed with the norm $\|k\|_{C_{\gamma}[0, T]}=\left\|t^{\gamma} k\right\|_{C[0, T]}$. Furthermore, we introduce

$$
K=\left\{k \in C^{1}(0, T] \mid k(t)>0, k^{\prime}(t)<0, t \in(0, T], k \in C_{\gamma}[0, T]\right\} .
$$

We assume that $u_{0} \in C^{2}(\bar{\Omega}), f \in C^{1}\left(\bar{Q}_{0, T}\right)$ and $g_{t} \in C^{2,1}\left(\bar{Q}_{0, T}\right)$ satisfy

$$
\begin{cases}A\left[u_{0}\right](x)+f(x, 0)=0, & x \in \Omega, \\ f_{t}(x, t)>0, & (x, t) \in Q_{0, T}, \\ g_{t}(x, t) \geq 0, & (x, t) \in \Sigma_{0, T} .\end{cases}
$$


Now we state our main result in this paper.

Theorem 1.1 Let $k \in K$ and (1.6) be held. Then the solution $(u, k)$ of the inverse problem (1.1)-(1.3) is unique.

Remark 1.1 Since $k$ is continuous with power singularity $t^{-\gamma}$, we will discuss the uniqueness of $k$ in the sense of $t^{\gamma} k_{1}(t)=t^{\gamma} k_{2}(t)$ for all $t \in[0, T]$, when $u\left(x_{0}, t ; k_{1}\right)=u\left(x_{0}, t ; k_{2}\right)$.

\section{Proof of Theorem 1.1}

In this section, the notations $Q_{\tau, T}, \Sigma_{\tau, T}$ and $L_{\tau, T}$ are similar to $Q_{0, T}, \Sigma_{0, T}$ and $L_{0, T}$ in Section 1, namely, $Q_{\tau, T}:=\Omega \times(\tau, T), \Sigma_{\tau, T}=\Gamma \times(\tau, T)$ and $L_{\tau, T}[u]:=u_{t}(x, t)-A[u](x, t)+$ $\int_{0}^{t} k(t-s) u_{t}(x, s) \mathrm{d} s$ in $Q_{\tau, T}$. In order to prove Theorem 1.1, we first give the following lemmas.

Lemma 2.1 Let $k \in K$ and $u \in C^{2,1}\left(\bar{Q}_{0, T}\right)$. If there exist $x_{1} \in \Omega$ and $t_{1} \in(0, T]$ such that $u$ attains the minimum value at $\left(x_{1}, t_{1}\right)$ on $\bar{Q}_{0, T}$, then we have

$$
\int_{\tau}^{t_{1}} k\left(t_{1}-s\right) u_{t}\left(x_{1}, s\right) \mathrm{d} s \leq 0, \quad \tau \in\left[0, t_{1}\right) .
$$

Proof Here we borrow the ideas used in dealing with the extremum principle of the Caputo derivative in [21]. Integration by parts yields

$$
\begin{array}{rl}
\int_{\tau}^{t_{1}} & k\left(t_{1}-s\right) u_{t}\left(x_{1}, s\right) \mathrm{d} s \\
= & \int_{\tau}^{t_{1}} k\left(t_{1}-s\right) \mathrm{d}\left[u\left(x_{1}, s\right)-u\left(x_{1}, t_{1}\right)\right] \\
= & \left.k\left(t_{1}-s\right)\left[u\left(x_{1}, s\right)-u\left(x_{1}, t_{1}\right)\right]\right|_{t=\tau} ^{t=t_{1}}-\int_{\tau}^{t_{1}}\left(k\left(t_{1}-s\right)\right)_{s}^{\prime}\left[u\left(x_{1}, s\right)-u\left(x_{1}, t_{1}\right)\right] \mathrm{d} s \\
= & \lim _{s \rightarrow t_{1}} k\left(t_{1}-s\right)\left[u\left(x_{1}, s\right)-u\left(x_{1}, t_{1}\right)\right]-k\left(t_{1}-\tau\right)\left[u\left(x_{1}, \tau\right)-u\left(x_{1}, t_{1}\right)\right] \\
& +\int_{\tau}^{t_{1}} k^{\prime}\left(t_{1}-s\right)\left[u\left(x_{1}, s\right)-u\left(x_{1}, t_{1}\right)\right] \mathrm{d} s .
\end{array}
$$

Since $k \in K$ and $u_{t} \in C\left(\bar{Q}_{\tau, T}\right)$, we have

$$
\begin{aligned}
& \lim _{s \rightarrow t_{1}} k\left(t_{1}-s\right)\left[u\left(x_{1}, s\right)-u\left(x_{1}, t_{1}\right)\right] \\
& \quad=-\lim _{s \rightarrow t_{1}}\left(t_{1}-s\right)^{\gamma} k\left(t_{1}-s\right) \frac{u\left(x_{1}, s\right)-u\left(x_{1}, t_{1}\right)}{s-t_{1}}\left(t_{1}-s\right)^{1-\gamma}=0 .
\end{aligned}
$$

Together with $k(t)>0, k^{\prime}(t)<0$ for $t \in(0, T]$ and $u\left(x_{1}, t_{1}\right)$ is the minimum value of $u$ on $\bar{Q}_{\tau, T}$, we obtain (2.1) from (2.2). This completes the proof of Lemma 2.1.

Lemma 2.2 Let $\tau \in[0, T]$ and $u \in C^{2,1}\left(\bar{Q}_{\tau, T}\right)$ satisfy $L_{\tau, t}[u](x, t) \geq 0$ in $Q_{\tau, T}$. Then we have

$$
\min _{\bar{Q}_{\tau, T}} u(x, t)=\min _{\partial_{p} Q_{\tau, T}} u(x, t),
$$

where $\partial_{p} Q_{\tau, T}:=(\bar{\Omega} \times\{t=\tau\}) \cup \Sigma_{\tau, T}$. 
Proof We first prove (2.3) when $L_{\tau, t}[u]>0$ in $Q_{\tau, T}$. Assume that (2.3) does not hold. Then there exist $x_{2} \in \Omega$ and $t_{2} \in(\tau, T]$ such that $u\left(x_{2}, t_{2}\right)=\min _{(x, t) \in \bar{Q}_{\tau, T}} u(x, t)$. Therefore $u_{t}\left(x_{2}, t_{2}\right) \leq 0$ and $A[u]\left(x_{2}, t_{2}\right) \geq 0$. Additionally, by Lemma 2.1 we have $\int_{\tau}^{t_{2}} k\left(t_{2}-\right.$ $s) u_{t}\left(x_{2}, s\right) \mathrm{d} s \leq 0$. Thus $L_{\tau, t}[u]\left(x_{2}, t_{2}\right) \leq 0$, which contradicts with $L_{\tau, t}[u](x, t)>0$ in $Q_{\tau, T}$.

Next we consider the general case of $L_{\tau, t}[u] \geq 0$ in $Q_{\tau, T}$. Let $v=u-\varepsilon e^{-t}$ with some $\varepsilon>0$. Then we have

$$
L_{\tau, t}[v]=L_{\tau, t}[u]+\varepsilon e^{-t}+\varepsilon \int_{\tau}^{t} k(t-s) e^{-s} \mathrm{~d} s>0 .
$$

According to the proved conclusion, it follows that

$$
\min _{\bar{Q}_{\tau, T}} u(x, t) \geq \min _{\bar{Q}_{\tau, T}} v(x, t)=\min _{\partial_{p} Q_{\tau, T}} v(x, t) \geq \min _{\partial_{p} Q_{\tau, T}} u(x, t)-\varepsilon e^{-\tau} .
$$

Letting $\varepsilon \rightarrow 0$, we get the desired conclusion and the proof is complete.

By using Lemma 2.2, we can prove the following lemma.

Lemma 2.3 Let (1.6) be held and $u$ be the solution of the problem (1.1)-(1.2). Then we have

$$
u_{t}(x, t)>0, \quad(x, t) \in Q_{0, T} .
$$

Proof By the equation of $u$ and (1.6), we have

$$
u_{t}(x, 0)=A\left[u_{0}\right](x)+f(x, 0)=0, \quad x \in \Omega .
$$

Letting $v=u_{t}$ and differentiating the equation in (1.1) with respect to $t$, we find that

$$
\begin{cases}L_{0, t}[v](x, t)=f_{t}(x, t), & (x, t) \in Q_{0, T}, \\ v(x, 0)=0, & x \in \Omega, \\ v(x, t)=g_{t}(x, t), & (x, t) \in \Sigma_{0, T},\end{cases}
$$

where we have used

$$
\left(\int_{0}^{t} k(t-s) u_{t}(x, s) \mathrm{d} s\right)_{t}=k(t) u_{t}(x, 0)+\int_{0}^{t} k(s) u_{t t}(x, t-s) \mathrm{d} s .
$$

According to Theorem A.1 in Appendix A, we have $v \in C^{2,1}\left(\bar{Q}_{0, T}\right)$ under $f_{t} \in C\left(\bar{Q}_{0, T}\right)$ and $g_{t} \in C^{2,1}\left(\bar{Q}_{0, T}\right)$. In addition, by $(1.6)$ we have $L_{0, t}[v](x, t) \geq 0$ in $Q_{0, T}$ and $\min _{\partial_{p} Q_{0, T}} v(x, t) \geq 0$. Then applying Lemma 2.2, we obtain

$$
v(x, t) \geq 0, \quad(x, t) \in \bar{Q}_{0, T} .
$$

Now we are ready to prove (2.6). If (2.6) does not hold, then $v$ attains the minimum value 0 on $\bar{Q}_{0, T}$ at $\left(x_{3}, t_{3}\right) \in Q_{0, T}$, and we have $v_{t}\left(x_{3}, t_{3}\right) \leq 0$ and $A v\left(x_{3}, t_{3}\right) \geq 0$. Additionally, by Lemma 2.1 we obtain $\int_{0}^{t_{3}} k\left(t_{3}-s\right) v_{t}\left(x_{3}, s\right) \mathrm{d} s \leq 0$. Therefore,

$$
0 \geq v_{t}\left(x_{3}, t_{3}\right)-A v\left(x_{3}, t_{3}\right)+\int_{0}^{t_{3}} k\left(t_{3}-s\right) v_{t}\left(x_{3}, s\right) \mathrm{d} s=f_{t}\left(x_{3}, t_{3}\right)
$$

This contradicts with $f_{t}(x, t)>0$ in $Q_{0, T}$. The proof of Lemma 2.3 is complete. 
Now we prove Theorem 1.1.

Proof of Theorem 1.1 Let $\left(u_{1}, k_{1}\right)$ and $\left(u_{2}, k_{2}\right)$ be two solutions of the inverse problem (1.1)(1.3). This implies that $\hat{u}:=u_{1}-u_{2}$ and $\hat{k}=k_{1}-k_{2}$ satisfy

$$
\begin{cases}L_{0, t}[\hat{u}](x, t)=-\int_{0}^{t} \hat{k}(t-s)\left(u_{2}\right)_{t}(x, s) \mathrm{d} s, & (x, t) \in Q_{0, T}, \\ \hat{u}(x, 0)=0, & x \in \Omega, \\ \hat{u}(x, t)=0, & (x, t) \in \Sigma_{0, T},\end{cases}
$$

and

$$
\hat{u}\left(x_{0}, t\right)=0, \quad t \in[0, T] .
$$

Here we have used $k_{1}$ as the kernel function in $L_{0, t}$ and this has no impact on the following proof. In order to prove the uniqueness of the inverse problem (1.1)-(1.3), it is sufficient to show that

$$
t^{\gamma} \hat{k}=0, \quad t \in[0, T] .
$$

Indeed, if (2.13) holds, then we have $\hat{k}(t)=0$ for $t \in(0, T]$, i.e. $\int_{0}^{t} \hat{k}(t-s)\left(u_{2}\right)_{t}(x, s) \mathrm{d} s=0$. Therefore, by Theorem 1.1, we obtain $\hat{u}(x, t)=0$ for $(x, t) \in \bar{Q}_{0, T}$.

We now prove (2.13) by contradiction. We assume that (2.13) does not hold and then set

$$
t_{0}=\inf \left\{t \in[0, T] \mid t^{\gamma} \hat{k}(t) \neq 0\right\} .
$$

Since $t^{\gamma} k_{1}, t^{\gamma} k_{2} \in C[0, T]$, there exists sufficiently small $\delta>0$ such that $t^{\gamma} k_{1}(t) \neq t^{\gamma} k_{2}(t)$ for $t \in\left[t_{0}, t_{0}+\delta\right]$. Without loss of generality, we can assume that $t^{\gamma} k_{1}(t)<t^{\gamma} k_{2}(t)$ for $t \in$ $\left[t_{0}, t_{0}+\delta\right]$. Next we prove

$$
\hat{u}(x, t)=0, \quad(x, t) \in \bar{Q}_{0, t_{0}} .
$$

Obviously, the conclusion is correct for $t_{0}=0$. When $t_{0}>0$, we consider the direct problem (1.1) and (1.2) in $Q_{0, t_{0}}$. By $\int_{0}^{t} \hat{k}(t-s)\left(u_{2}\right)_{t}(x, s) \mathrm{d} s=0$ in $\left[0, t_{0}\right]$ and Theorem A.1 we obtain $\|\hat{u}\|_{C^{2,1}\left(\bar{Q}_{0, t_{0}}\right)}=0$. Therefore, $\hat{u}(x, t)=0$ for $(x, t) \in \bar{Q}_{0, t_{0}}$.

Now on the basis of (2.11), (2.14), and (2.15), we have

$$
\begin{cases}L_{t_{0}, t}[\hat{u}](x, t)=-\int_{t_{0}}^{t} \hat{k}(t-s)\left(u_{2}\right)_{t}(x, s) \mathrm{d} s, & (x, t) \in Q_{t_{0}, t_{0}+\delta}, \\ \hat{u}\left(x, t_{0}\right)=0, & x \in \Omega, \\ \hat{u}(x, t)=0, & (x, t) \in \Sigma_{t_{0}, t_{0}+\delta} .\end{cases}
$$

By Lemma 2.3, we have $\left(u_{2}\right)_{t}(x, t)>0$ for $(x, t) \in Q_{t_{0}, t_{0}+\delta}$. Therefore, $L_{t_{0}, t}[\hat{u}](x, t)>0$ in $Q_{t_{0}, t_{0}+\delta}$. Applying Lemma 2.2, we have $\hat{u}(x, t) \geq 0$ in $\bar{Q}_{t_{0}, t_{0}+\delta}$. Furthermore, we can obtain

$$
\hat{u}(x, t)>0, \quad(x, t) \in Q_{t_{0}, t_{0}+\delta} .
$$

Otherwise, there exists $\left(x_{4}, t_{4}\right) \in Q_{t_{0}, t_{0}+\delta}$ such that $\hat{u}\left(x_{4}, t_{4}\right)$ is the minimum value of $\hat{u}$ on $\bar{Q}_{t_{0}, t_{0}+\delta}$. Then we have $\hat{u}\left(x_{4}, t_{4}\right)_{t}=0$ and $A u\left(x_{4}, t_{4}\right) \geq 0$. Additionally, Lemma 2.1 gives 
$\int_{t_{0}}^{t_{4}} k_{1}\left(t_{4}-s\right) \hat{u}_{t}(x, s) \mathrm{d} s \leq 0$ and we find that

$$
0 \geq \hat{u}\left(x_{4}, t_{4}\right)_{t}-A u\left(x_{4}, t_{4}\right)+\int_{t_{0}}^{t_{4}} k_{1}\left(t_{4}-s\right) \hat{u}_{t}\left(x_{4}, s\right) \mathrm{d} s=L_{t_{0}, t}[\hat{u}]\left(x_{4}, t_{4}\right)
$$

which contradicts with $L_{t_{0}, t}[\hat{u}](x, t)>0$ in $Q_{t_{0}, t_{0}+\delta}$. Thus (2.17) follows. However, by (2.12), $\hat{u}\left(x_{0}, t\right)=0$ for $x_{0} \in \Omega$ and $t \in\left(t_{0}, t_{0}+\delta\right)$. This is a contradiction. Thus the proof of Theorem 1.1 is complete.

\section{Conclusion}

In this paper we study an inverse weakly singular memory kernel problem for an integrodifferential equation related to the Basset problem. In order to determine the weakly singular term, we only use the measurement data at a fixed point $x_{0} \in \Omega$, rather than the usual measurement data on the whole or part of $\Omega$ in previous studies of inverse kernel problems. The uniqueness of our inverse problem is shown by using a maximum principle related to an integro-differential operator. In addition, the existence and uniqueness of the direct Basset problem with general kernel function are also given, which extends the results in [1] and [2].

\section{Appendix A}

Here, we study the existence and uniqueness of the following direct problem:

$$
\begin{cases}L_{0, t}[u](x, t)=f(x, t), & (x, t) \in Q_{0, T} \\ u(x, 0)=u_{0}(x), & x \in \Omega, \\ u(x, t)=g(x, t), & (x, t) \in \Sigma_{0, T}\end{cases}
$$

We can prove the following.

Theorem A.1 Let $k \in C_{\gamma}[0, T]$ with some $\gamma \in(0,1), u_{0} \in C^{2}(\bar{\Omega}), f \in C\left(\bar{Q}_{0, T}\right)$ and $g \in$ $C^{2,1}\left(\bar{Q}_{0, T}\right)$. Then the direct problem (A.1) has a unique solution $u \in C^{2,1}\left(\bar{Q}_{0, T}\right)$ such that

$$
\begin{aligned}
& \|u\|_{C^{2,1}\left(\bar{Q}_{0, t}\right)} \\
& \quad \leq C\left(\left\|u_{0}\right\|_{C^{2}(\bar{\Omega})}+\|f\|_{C\left(\bar{Q}_{0, t}\right)}+\|g\|_{C^{2,1}\left(\bar{Q}_{0, t}\right)}\right) E_{1-\gamma}\left(\|k\|_{C_{\gamma}[0, T]} \Gamma(1-\gamma) t^{1-\gamma}\right),
\end{aligned}
$$

where $E_{1-\gamma}(z)$ is the Mittag-Leffler function defined by $E_{1-\gamma}(z)=\sum_{n=0}^{\infty} \frac{z^{n}}{\Gamma(n(1-\gamma)+1)}$.

Remark A.1 When $k=\frac{1}{\Gamma(1-\alpha)} t^{-\alpha}$ with $0<\alpha<1$, the equation of $u$ can be rewritten as

$$
u_{t}(x, t)-A[u](x, t)+\partial_{t}^{\alpha} u(x, t)=f(x, t), \quad(x, t) \in Q_{0, T} .
$$

This is a time fractional parabolic equation to describe the Basset problem [6].

Proof We will use a fixed point argument to prove this theorem. To do this, we set

$$
\begin{aligned}
V_{r}= & \left\{v \in C^{2,1}\left(\bar{Q}_{0, T}\right) \mid v(x, 0)=u_{0}(x), x \in \Omega, v(x, t)=g(x, t),(x, t) \in \Sigma_{0, T},\right. \\
& \left.\|v\|_{C^{2,1}\left(\bar{Q}_{0, T}\right)} \leq r\right\}
\end{aligned}
$$


with some $r>0$, which will be specified below. For given $v \in V_{r}$, we consider

$$
\begin{cases}u_{t}(x, t)-A[u](x, t)=G[v](x, t)+f(x, t), & (x, t) \in Q_{0, T}, \\ u(x, 0)=u_{0}(x), & x \in \Omega, \\ u(x, t)=g(x, t), & (x, t) \in \Sigma_{0, T},\end{cases}
$$

with $G[v](x, t)=-\int_{0}^{t} k(t-s) v(x, s) \mathrm{d} s$. The standard result for linear parabolic equation [22] shows that there exists a unique solution $u \in C^{2,1}\left(\bar{Q}_{0, T}\right)$ of the problem (A.4) such that

$$
\|u\|_{C^{2,1}\left(\bar{Q}_{0, t}\right)} \leq C\left(\left\|u_{0}\right\|_{C^{2}(\bar{\Omega})}+\|G[v]+f\|_{C\left(\bar{Q}_{0, t}\right)}+\|g\|_{C^{2,1}\left(\bar{Q}_{0, t}\right)}\right), \quad 0 \leq t \leq T .
$$

Therefore, the following mapping:

$$
\Phi: V_{r} \rightarrow C^{2,1}\left(\bar{Q}_{0, T}\right), \quad v \mapsto u
$$

is well defined.

We want to choose $T$ small enough to prove $\Phi$ is a contraction on $V_{r}$, which implies that $\Phi$ has a unique fixed point $u$ in $V_{r}$. By $k \in C_{\gamma}[0, T]$, we have

$$
\begin{aligned}
\|G[v]\|_{C\left(\bar{Q}_{0, t}\right)} & \leq \int_{0}^{t} k(t-s)\|v\|_{C^{2,1}\left(\bar{Q}_{0, s}\right)} \mathrm{d} s \\
& \leq C\|k\|_{C_{\gamma}[0, T]} \int_{0}^{t}(t-s)^{-\gamma}\|v\|_{C^{2,1}\left(\bar{Q}_{0, s}\right)} \mathrm{d} s .
\end{aligned}
$$

Substituting (A.7) into (A.5) yields

$$
\|u\|_{C^{2,1}\left(\bar{Q}_{0, t}\right)} \leq C\left(\left\|u_{0}\right\|_{C^{2}(\bar{\Omega})}+\|f\|_{C\left(\bar{Q}_{0, T}\right)}+\|g\|_{C^{2,1}\left(\bar{Q}_{0, T}\right)}\right)+\frac{C t^{1-\gamma}}{1-\gamma}\|k\|_{C_{\gamma}[0, T]} r .
$$

We fix

$$
2 r=C\left(\left\|u_{0}\right\|_{C^{2}(\bar{\Omega})}+\|f\|_{C\left(\bar{Q}_{0, T}\right)}+\|g\|_{C^{2,1}\left(\bar{Q}_{0, T}\right)}\right) .
$$

Then we can choose $T_{1}$ to satisfy

$$
\|u\|_{C^{2,1}\left(\bar{Q}_{0, T}\right)} \leq r
$$

for all $T \in\left[0, T_{1}\right]$, and from which it follows that $\Phi\left(V_{r}\right) \subset V_{r}$. On the other hand, given $v_{1}, v_{2} \in V_{r}, U:=\Phi\left(v_{1}\right)-\Phi\left(v_{2}\right)$ satisfies

$$
\begin{cases}U_{t}(x, t)-A[U](x, t)=G\left[v_{1}\right](x, t)-G\left[v_{2}\right](x, t), & (x, t) \in Q_{0, T}, \\ U(x, 0)=0, & x \in \Omega, \\ U(x, t)=0, & (x, t) \in \Sigma_{0, T} .\end{cases}
$$

Therefore, applying (A.5) we have

$$
\|U\|_{C^{2,1}\left(\bar{Q}_{0, t}\right)} \leq C\left(\left\|G\left[v_{1}\right]-G\left[v_{2}\right]\right\|_{C\left(\bar{Q}_{0, t}\right)}\right) \leq \frac{C t^{1-\gamma}}{1-\gamma}\|k\|_{C_{\gamma}[0, T]}\left\|v_{1}-v_{2}\right\|_{C^{2,1}\left(\bar{Q}_{0, t}\right)}
$$


for all $t \in[0, T]$. Then there exists sufficiently small $T_{2}$ such that

$$
\left\|\Phi\left(v_{1}\right)-\Phi\left(v_{2}\right)\right\|_{C^{2,1}\left(\bar{Q}_{0, T}\right)} \leq 1 / 2\left\|v_{1}-v_{2}\right\|_{C^{2,1}\left(\bar{Q}_{0, T}\right)}
$$

for all $T \in\left[0, T_{2}\right]$. By (A.10) and (A.13), we find that $\Phi: V_{r} \rightarrow V_{r}$ is a contraction mapping for $T \leq \min \left\{T_{1}, T_{2}\right\}$. Thus there exists a local unique solution $u \in C^{2,1}\left(\bar{Q}_{0, T}\right)$ of the problem (A.1) for sufficiently small $T$.

In order to obtain the global existence, it is sufficient to prove that the solution $u$ of the problem (A.1) satisfies

$$
\|u\|_{C^{2,1}\left(\bar{Q}_{0, T}\right)}<\infty
$$

for any $T$. Indeed, if (A.14) holds, then we can extend the local solution repeatedly to the whole interval $[0, T]$ by the above fixed point arguments. By the estimate of Schauder type for parabolic equation [23], we find that for any $t \in[0, T]$,

$$
\begin{aligned}
\|u\|_{C^{2,1}\left(\bar{Q}_{0, t}\right)} \leq & C\left(\left\|u_{0}\right\|_{C^{2}(\bar{\Omega})}+\|G[u]+f\|_{C\left(\bar{Q}_{0, t}\right)}+\|g\|_{C^{2,1}\left(\bar{Q}_{0, t}\right)}\right) \\
\leq & C\left(\left\|u_{0}\right\|_{C^{2}(\bar{\Omega})}+\|f\|_{C\left(\bar{Q}_{0, t}\right)}+\|g\|_{C^{2,1}\left(\bar{Q}_{0, t}\right)}\right) \\
& +C\|k\|_{C_{\gamma}[0, T]} \int_{0}^{t}(t-s)^{-\gamma}\|u\|_{C^{2,1}\left(\bar{Q}_{0, s}\right)} \mathrm{d} s,
\end{aligned}
$$

which implies (A.2) by the weakly singular Gronwall inequalities [24]. Since $E_{1-\gamma}(z)$ is continuous on $[0, T]$, (A.14) holds for any $T$. This completes the proof of Theorem A.1.

\section{Competing interests}

The authors declare that they have no competing interests.

\section{Authors' contributions}

All authors contributed equally to the writing of this paper. All authors read and approved the final manuscript.

\section{Author details}

'School of Mathematics and Statistics, Nanjing University of Information Science and Technology, Nanjing, 210044, China. ${ }^{2}$ Department of Mathematics and Statistics, The University of Vermont, Burlington, 05401, USA.

\section{Acknowledgements}

The first author is supported by NSFC (No. 11201238). This work has been completed while the first author visited Department of Mathematics and Statistics at The University of Vermont and he acknowledges the hospitality and support of the Department and the University.

Received: 8 June 2014 Accepted: 1 October 2014 Published online: 15 October 2014

\section{References}

1. Ashyralyev, A: Well-posedness of fractional parabolic equations. Bound. Value Probl. 2013, Article ID 31 (2013). doi:10.1186/1687-2770-2013-31

2. Ashyralyev, A: Well-posedness of the Basset problem in spaces of smooth functions. Appl. Math. Lett. 24, 1176-1180 (2011)

3. Li, F, Liang, J, Xu, HK: Existence of mild solutions for fractional integrodifferential equations of Sobolev type with nonlocal conditions. J. Math. Anal. Appl. 391, 510-525 (2012)

4. Kilbas, AA, Srivastava, HM, Trujillo, JJ: Theory and Applications of Fractional Differential Equations. Elsevier, Amsterdam (2006)

5. Podlubny, I: Fractional Differential Equations. Academic Press, San Diego (1999)

6. Basset, AB: On the descent of a sphere in a viscous liquid. Q. J. Math. 42, 369-381 (1910)

7. Cakir, Z: Stability of difference schemes for fractional parabolic PDE with the Dirichlet-Neumann conditions. Abstr. Appl. Anal. 2012, Article ID 463746 (2012)

8. Ashyralyev, A, Cakir, Z: On the numerical solution of fractional parabolic partial differential equations with the Dirichlet condition. Discrete Dyn. Nat. Soc. 2012, Article ID 696179 (2012) 
9. Ashyralyev, A: Well-posedness of parabolic differential and difference equations with the fractional differential operator. Malaysian J. Math. Sci. 6, suppl., 73-89 (2012)

10. Ashyralyev, A, Cakir, Z: FDM for fractional parabolic equations with the Neumann condition. Adv. Differ. Equ. 2013, Article ID 120 (2013). doi:10.1186/1687-1847-2013-120

11. Ashyralyev, A, Cakir, Z: r-Modified Crank-Nicholson difference scheme for fractional parabolic PDE. Bound. Value Probl. 2014, Article ID 76 (2014). doi:10.1186/1687-2770-2014-76

12. Ashyralyev, A, Emirov, N, Cakir, Z: Well-posedness of fractional parabolic differential and difference equations with Dirichlet-Neumann conditions. Electron. J. Differ. Equ. 2014, 97 (2014)

13. Liu, W, Chen, K: The functional variable method for finding exact solutions of some nonlinear time-fractional differential equations. Pramana 81, 377-384 (2013)

14. Saedpanah, F: Well-posedness of an integro-differential equation with positive type kernels modeling fractional order viscoelasticity. Eur. J. Mech. A, Solids 44, 201-211 (2014)

15. Wheeler, MF: A priori $L_{2}$ error estimates for Galerkin approximations to parabolic partial differential equations. SIAM J. Numer. Anal. 10, $723-759$ (1973)

16. Colombo, F, Guidetti, D: A global in time existence and uniqueness result for a semilinear integrodifferential parabolic inverse problem in Sobolev spaces. Math. Models Methods Appl. Sci. 17, 537-565 (2007)

17. Colombo, F, Guidetti, D: Identification of the memory kernel in the strongly damped wave equation by a flux condition. Commun. Pure Appl. Anal. 8, 601-620 (2009)

18. Lorenzi, A, Rocca, E: Identification of two memory kernels in a fully hyperbolic phase-field system. J. Inverse III-Posed Probl. 16, 147-174 (2008)

19. $\mathrm{Wu}, \mathrm{B}, \mathrm{Liu}, \mathrm{J}: \mathrm{A}$ global in time existence and uniqueness result for an integrodifferential hyperbolic inverse problem with memory effect. J. Math. Anal. Appl. 373, 585-604 (2011)

20. Janno, J, Von Wolfersdorf, L: Identification of weakly singular memory kernels in heat conduction. Z. Angew. Math. Mech. 77, 243-257 (1997)

21. Luchko, Y: Maximum principle for the generalized time-fractional diffusion equation. J. Math. Anal. Appl. 351, 218-223 (2009)

22. Wu, Z, Yin, J, Wang, C: Elliptic and Parabolic Equations. World Scientific, Singapore (2006)

23. Friedman, A: Partial Differential Equations of Parabolic Type. Holt, Rinehart \& Winston, New York (1964)

24. Dixo, J, Mckee, S: Weakly singular discrete Gronwall inequalities. Z. Angew. Math. Mech. 66, 535-544 (1986)

doi:10.1186/s13661-014-0229-9

Cite this article as: Wu and Yu: Uniqueness of an inverse problem for an integro-differential equation related to the Basset problem. Boundary Value Problems 2014 2014:229.

\section{Submit your manuscript to a SpringerOpen ${ }^{\circ}$ journal and benefit from:}

- Convenient online submission

Rigorous peer review

- Immediate publication on acceptance

- Open access: articles freely available online

- High visibility within the field

- Retaining the copyright to your article 